\title{
On the Reconfiguration Algorithm for Fault-Tolerant VLSI Arrays
}

\author{
Jigang Wu and Srikanthan Thambipillai \\ Centre for High Performance Embedded Systems \\ Nanyang Technological University, Singapore, 639798 \\ \{asjgwu, astsrikan\}@ntu.edu.sg
}

\begin{abstract}
In this paper, an improved algorithm is presented for the NP-complete problem of reconfiguring a two-dimensional degradable VLSI array under the row and column routing constraints. The proposed algorithm adopts the partial computing for the logical row exclusion so that the most efficient algorithm, cited in literature, is speeded up without loss of performance. In addition, a flaw in the earlier approach is also addressed. Experimental results show that our algorithm is approximately $50 \%$ faster than the above stated algorithm.
\end{abstract}

Keywords: degradable VLSI array, reconfiguration, fault-tolerance, greedy algorithm, NP-completeness

\section{Introduction}

The mesh-connected processor array has a regular and modular structure and allows fast implementation of most signal and image processing algorithms. With the advancement in VLSI (very large scale integration) and WSI (wafer scale integration) technologies, integrated systems for mesh-connected processor arrays can now be built on a single chip or wafer. As the density of VLSI and WSI arrays increases, the probability of the occurrence of defects in the arrays during fabrication also increases. These defects obviously affect the reliability of the whole system. Thus, fault-tolerant technologies must be employed to enhance the yield and reliability of VLSI/WSI arrays.

There are generally two methods for reconfiguration in fault-tolerant technologies, namely, the redundancy approach and the degradation approach. Various strategies to restructure a faulty physical system using the redundancy approach are described in many papers, e.g.,[1-12]. Degradation approach uses as many fault-free elements as possible to construct a target system. The final dimension is flexible and depends on the needs of the application. Usually, a maximum dimension is desirable. Literatures [13-15] have studied the problem of reconfiguring two-dimensional degradable arrays. They have shown that most problems that arise under the constraint row and column rerouting are NP-complete.

In this paper, we consider the reconfiguration problem of two-dimensional degradable VLSI/WSI arrays. It is defined as follows [13-15]. 
Given an $m \times n$ mesh-connected host array $H$ with the fault density $\rho(0 \leq$ $\rho<1$ ), integers $r$ and $c$, find a $m^{\prime} \times n^{\prime}$ fault-free subarray $T$ under the row and column rerouting scheme such that $m^{\prime} \geq r$ and $n^{\prime} \geq c$.

The latest work for this problem is the algorithm in [15], which is denoted as RCRoute in this paper. This algorithm is dominated by two sub-procedures named Logical_Row_Exclusion (LRE) and Greedy_Column_Rerouting (GCR), respectively. The time complexity of each sub-procedure is $O((1-\rho) \cdot m \cdot n)$. The routing manner in GCR leads to reconfiguring two neighboring fault-free elements lying in same physical row into the same logical column. But the related architecture does not support this kind of the routing. In this paper we point out this flaw and repair it. In addition, we also present a partial computing approach for the logical row exclusion. The new approach reduces the time complexity of $L R E$ from $O((1-\rho) \cdot m \cdot n)$ into $O((1-\rho) \cdot n)$. Thus, we improve RCRoute in running time, without loss of performance. Experimental results show that the improved algorithm is approximately $50 \%$ faster than RCRoute.

\section{Preliminaries}

This section gives the definitions and the notations used in this paper.

In this paper, the original VLSI/WSI array that has been manufactured is called a host array. This host array may contain faulty elements. A degradable subarray of the host array, which contains no faulty element, is called a target array or logical array. The rows (columns) in the host array and target array are called physical rows (columns) and logical rows (columns), respectively. row $(e)$ $(\operatorname{col}(e))$ denotes the physical row (column) index of element $e . H(S)$ denotes the host (logical) array. $R_{i}$ denotes the $i$ th logical row. Using the same assumptions as in [13][14][15], in this paper two neighboring elements in the host array are connected by a four-port switch. All switches and links in an array are assumed to be fault-free since they have very simple structure.

In a host array, if $e(i, j+1)$ is a faulty element, then $e(i, j)$ can communicate with $e(i, j+2)$ directly and data will bypass $e(i, j+1)$. This scheme is called row bypass scheme. If $e(i, j)$ can connect directly to $e\left(i^{\prime}, j+1\right)$ with external switches, where $\left|i^{\prime}-i\right| \leq d$, this scheme is called row rerouting scheme, $d$ is called row compensation distance. The column bypass scheme and the column rerouting scheme can be defined similarly. By limiting the compensation distance to 1, we essentially localize the movements of reconfiguration in order to avoid complex reconfiguration algorithm. In all figures of this paper, the shaded boxes stand for faulty elements and the white ones stand for the fault-free elements.

\section{Algorithms}

In this section we point out a flaw in RCRoute[15] and repair it. Then we present our algorithm denoted $N e w_{-} R C R o u t e$ in this paper. 


\section{$3.1 \quad$ Updating RCRoute}

For the row and column rerouting scheme, the latest efficient work is the algorithm RCRoute[15]. This greedy algorithm consists of two procedures namely Row_First and Column_First. Column_First is invoked after running Row_First. Column_First is identical to Row_First except that the roles of rows and columns are interchanged. Therefore, Row_First plays a key role in the description of RCRoute. For the detail description of Row_First, see [15].

There are two key sub-procedures in Row_First, denoted as LRE and GCR. The sub-procedure LRE selects one row to be excluded from the set that was previously selected and uses it to compensate for faulty elements in its two neighboring rows. Let $M_{i}$ denote the maximum number of logical columns that pass through two consecutive rows $R_{i}$ and $R_{i+1}$, where $i \in\{0,1, \ldots, k-1\}$. Let $M_{\gamma}=\min _{0 \leq i \leq k-1} M_{i}$. LRE first calculates $M_{\gamma}$ and selects the row $R_{\gamma}$, and then decides whether $R_{\gamma}$ or $R_{\gamma+1}$ will be excluded. Let $X$ denote the maximum number of logical columns that pass through $R_{\gamma-1}$ and $R_{\gamma+1}$. Let $Y$ denote the maximum number of logical columns that pass through $R_{\gamma}$ and $R_{\gamma+2}$. If $X>Y$, then row $R_{\gamma}$ is selected for exclusion, otherwise, $R_{\gamma+1}$ is excluded.

$G C R$ is used for finding a target array that contains a set of selected logical rows. It reroutes the fault-free elements to form logical columns. The successor of the fault free element $u$ in $R_{i}$ is selected from $\operatorname{Adj}(u)$ in a left-to-right manner, where $\operatorname{Adj}(u)=\left\{v: v \in R_{i+1}, v\right.$ is fault-free, $\left.\quad|\operatorname{col}(u)-\operatorname{col}(v)| \leq 1\right\}$. For the detailed description of the procedure, see $[14,15]$.

The sub-procedures $L R E$ and GCR are executed iteratively until the rowbased target array is found. LRE tests $O((1-\rho) \cdot m \cdot n)$ valid interconnections in the $m \times n$ host array in a row by row fashion, and GCR tests these valid interconnections column by column. Obviously, they have same time complexity $O((1-\rho) \cdot m \cdot n)$.

As can be seen from [15], the four-port switch model has a very simple architecture. But it is due to this simple construction that provides less functions that the switch model does not support reconfiguring two neighboring faultfree elements lying in same row into same logical column. Assume $R_{\gamma}$ is to be excluded by subprocedure $L R E$. Then $R_{\gamma}$ will be used to compensate for faulty elements in its two neighboring rows, $R_{\gamma-1}$ and $R_{\gamma+1}$, i.e., each fault-free element $e(\gamma, j), 1 \leq j \leq n$, will be used to compensate $e(\gamma-1, j)$ or $e(\gamma+1, j)$ if they are faulty. After compensation, The subprocedure $G C R$ will be executed on $\left\{R_{1}, R_{2}, \cdots, R_{\gamma-1}, R_{\gamma+1}, \cdots, R_{m}\right\}$ to find the current target array. However, it is possible that two neighboring fault-free elements in $R_{\gamma}$ will be rerouted into same logical column by GCR. That is the flaw of RCRoute.

We correct RCRoute by adding the constraint $\operatorname{row}(u)<\operatorname{row}(v)$ into the definition of set $\operatorname{Adj}(u)$, i.e., let $\operatorname{Adj}(u)=\left\{v: v \in R_{i+1}, v\right.$ is fault-free, $|\operatorname{col}(u)-\operatorname{col}(v)| \leq 1$ and $\operatorname{row}(u)<\operatorname{row}(v)\}$. The constraint limits the elements in the result logical column, in the strictly increasing order of their physical row indices. This prevents the above conflict when GCR runs. 


\subsection{Partial Computing for Logical Row Exclusion}

Assume $R_{1}, R_{2}, \ldots, R_{k}$ (initially, $k=m$ ) are the previously selected logical rows. In order to select one row, say $R_{l}$, to be excluded in the current iteration, $L R E$ takes $O((1-\rho) \cdot k \cdot n)$ time to calculate $M_{1}, M_{2}, \ldots, M_{k-1}$ and takes $O(k)$ to select the minimum $M_{\gamma}$ resulting in $l=\gamma$ or $\gamma+1$ according to the compensation approach. In Row_First[15], LRE does not reuse the previous calculation of $M_{i}, 1 \leq i<k$. In fact, except for $M_{l-2}, M_{l}$ and $M_{l+1}$, these $M_{i}$ calculated in the previous iteration are also available in the current iteration as the compensations by $R_{l}$ only affect $R_{l-1}$ and $R_{l+1}$. We only need to update $M_{l-2}, M_{l}$ and $M_{l+1}$. Hence, we simplify the sub-procedure $L R E$ into New_LRE (Fig. 1 (left)) in order to avoid the repeat calculations of $M_{i}$ except for $M_{l-2}, M_{l}$ and $M_{l+1}$. The initial values of each $M_{i}$ can be calculated out of the body of while-loop in Row_First [15]. Obviously, this simplified approach saves the running time of RCRoute but does not affect its harvest since the strategy for the selection of the row to be excluded has not been changed. By simple analysis, the running time of LRE in $q$ th iteration of Row_First is reduced from $O((1-\rho) \cdot q \cdot n)$ to $O((1-\rho) \cdot n)$ since only $M_{l-2}, M_{l}$ and $M_{l+1}$ need to be updated, where $1 \leq q \leq m$. In the other hand, New_LRE still uses the same compensation strategies as described in RCRoute[15] after the row $R_{l}$ is excluded. The time complexity of the compensation is $O((1-\rho) \cdot n)$. Hence, the time complexity of New $L R E$ is $O((1-\rho) \cdot n)$, which is far lower than $O((1-\rho) \cdot m \cdot n)$, the time complexity of LRE. Figure 1 shows the formal description of the improved algorithm.
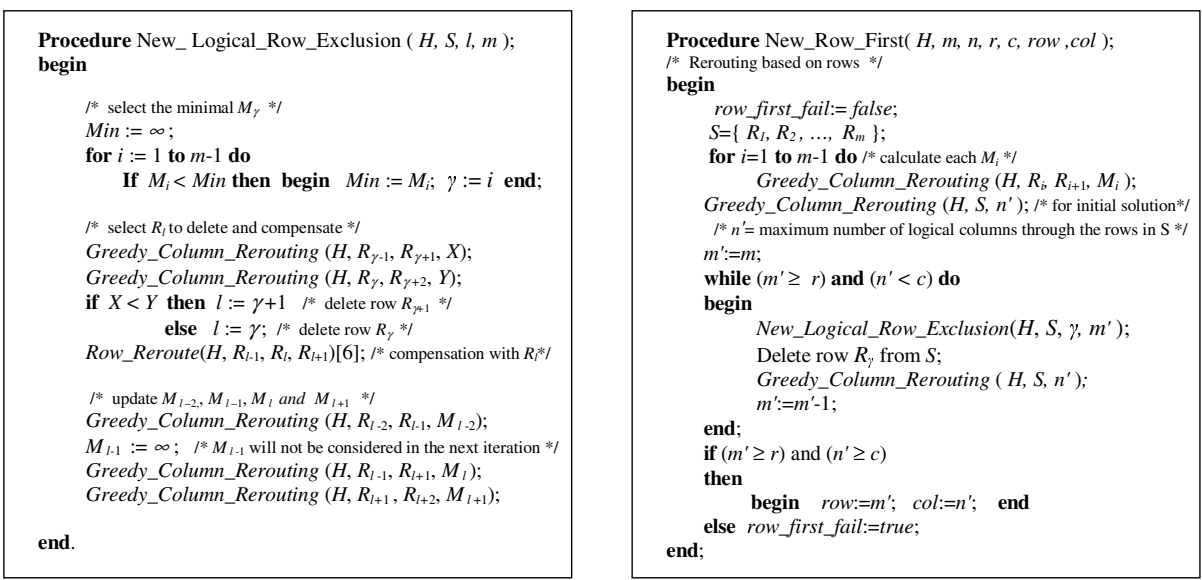

Fig. 1. The formal description of the procedure $N_{w}{ }_{-} L R E$ and New_Row_First 


\subsection{Main Algorithm and Complexity}

For the description of the main algorithm, we first describe a procedure called New_Row_First, which is used to find a target array with maximum size based on the row. Let $m^{\prime}$ be the number of logical rows and $n^{\prime}$ be the number of logical columns of the target array. The current logical array is $S=\left\{R_{1}, R_{2}, \cdots, R_{m^{\prime}}\right\}$. Initially, all rows in the host array are selected for inclusion into the target array. Thus, each logical row in $S$ is also a physical row. The formal description of New_Row_First is shown in Fig. 1 (right).

In New_Row_First, the code before the while-loop initializes the data structures, calculates all $M_{i}$ and gets the initial target array. The running time needed by all these steps is bounded by $O((1-\rho) \cdot m \cdot n)$. In the while-loop, New_LRE runs in $O((1-\rho) \cdot n)$ and $G C R$ runs in $O((1-\rho) \cdot m \cdot n)$. Hence, the while-loop runs in $O((m-r) \cdot(1-\rho) \cdot m \cdot n)$, i.e., the time complexity of New_Row_First is $O((m-r) \cdot(1-\rho) \cdot m \cdot n)$.

Similarly, we can describe a procedure New_Column_First to find a target array with maximum size based on the column. Its time complexity is $O((n-$ $c) \cdot(1-\rho) \cdot m \cdot n)$.

The structure of the main algorithm, denoted as New_RCRoute, is the same as that of RCRoute. but the subprocedures have been improved. The largest array derived from procedures New_Row_First and New_Column_First is taken as the target array for $H$. The time complexity of algorithm New_RCRoute is $O(\max \{(m-r),(n-c)\} \cdot(1-\rho) \cdot m \cdot n)$, which is the lower bound of the complexity for row and column rerouting[15].

\section{Experimental Results}

We report our experimental results in this section. We have implemented the algorithm RCRoute (corrected version) and the improved algorithm New_RCRoute in C on a personal computer-Intel Pentium-III $500 \mathrm{MHZ}$. The implementations of the two algorithms are modified accordingly to find maximal target arrays and maximal square target arrays. In our experiments, harvest and degradation, formulated in [13],[14] and [15], are calculated for each target array. In order to make a fair comparison between New_RCRoute and RCRoute, we keep the same assumptions as in [14],[15], i.e., the faults in random host arrays were generated by a uniform random generator; The fault size in host array is from $0.1 \%$ to $10 \%$ for the experiments. Both algorithms are tested with the same random input instances. The running time and the size of each target array obtained by New_RCRoute is compared with the corresponding array obtained by RCRoute[15]. Table 1 summarizes the experimental results for the random host arrays with different sizes. The improvement in running time is calculated by

$$
\left(1-\frac{\text { running_time_of_New_RCRoute }}{\text { running_time_of_RCRoute }}\right) \times 100 \% \text {. }
$$

The calculations required to arrive at solutions for maximal target array encompass the solution for maximal square target array. Hence, without loss of 
Table 1. The comparison of running time for 20 random instances

\begin{tabular}{|c|rr||c|c|c|c||c|}
\hline \multicolumn{2}{|c|}{} & \multicolumn{2}{|c|}{ Performance } & \multicolumn{3}{c|}{ Running Time } \\
\hline Array & $\begin{array}{c}\text { Fault } \\
\text { Size }\end{array}$ & $(\%)$ & $\begin{array}{c}\text { Harvest } \\
(\%)\end{array}$ & $\begin{array}{c}\text { Degrad. } \\
(\%)\end{array}$ & $\begin{array}{c}\text { RCRoute } \\
(\mathrm{s})\end{array}$ & $\begin{array}{c}\text { New_RCRoute } \\
(\mathrm{s})\end{array}$ & $\begin{array}{c}\text { Improve } \\
(\%)\end{array}$ \\
\hline $64 \times 64$ & 4 & 0.1 & 98.53 & 1.56 & 0.296 & 0.150 & 49.3 \\
$64 \times 64$ & 40 & 1.0 & 96.29 & 4.65 & 0.296 & 0.149 & 49.7 \\
$64 \times 64$ & 409 & 10.0 & 84.52 & 23.91 & 0.286 & 0.143 & 50.0 \\
& & & & & & & \\
$128 \times 128$ & 16 & 0.1 & 98.85 & 1.24 & 2.313 & 1.191 & 48.5 \\
$128 \times 128$ & 163 & 1.0 & 97.15 & 3.82 & 2.307 & 1.189 & 48.5 \\
$128 \times 128$ & 1638 & 10.0 & 84.61 & 23.84 & 2.213 & 1.152 & 47.9 \\
& & & & & & & \\
$256 \times 256$ & 65 & 0.1 & 99.24 & 0.86 & 18.316 & 9.441 & 48.5 \\
$256 \times 256$ & 655 & 1.0 & 97.56 & 3.41 & 18.160 & 9.369 & 48.4 \\
$256 \times 256$ & 6553 & 10.0 & 84.37 & 24.07 & 17.340 & & \\
& & & & & & & \\
$512 \times 512$ & 262 & 0.1 & 99.41 & 0.69 & 147.291 & 76.148 & 47.7 \\
$512 \times 512$ & 2621 & 1.0 & 97.92 & 3.06 & 145.004 & 75.081 & 48.3 \\
$512 \times 512$ & 26214 & 10.0 & 84.89 & 23.60 & 137.259 & 72.033 & 47.5 \\
\hline
\end{tabular}

generality, we collect the running time only in the case of finding maximal target array. Table 1 shows the running time comparisons for the maximal target array. For each random instance, the running time required by New_RCRoute is significantly less than that required by RCRoute. For example, for the host array of size $256 \times 256$ with 655 fault elements, the running time for New_RCRoute is 9.369 seconds, while it is 18.160 seconds for RCRoute. The improvement in running time is $48.4 \%$, which is nearly equal to $50 \%$. Except for small size instances such as $64 \times 64$, increase in fault density in the host array leads to less improvement in running time as more backtracking is needed in routing. For instance, for the $512 \times 512$ host array with the 26214 fault elements, the improvement in running time is $47.5 \%$, which is a little less than $48.3 \%$, the improvement running time for the $512 \times 512$ host array with the 262 fault elements.

We can conclude from the analysis above that our algorithm New_RCRoute reduced the running time by approximately 50\%, especially, for low density of faults in the host arrays, without loss of harvest.

\section{Conclusions}

We have presented a degradation approach for the reconfiguration in VLSI/WSI arrays under the rerouting constraint row and column rerouting. We have proposed a new strategy for the row selection. The new strategy binds well for highspeed realizations. For different sized host arrays, our algorithm has maintained harvest and degradation while reducing the running time by approximately $50 \%$. Method to overcome the flaw in one of the recent contributions in this area was also made. The improved algorithms have been implemented and experimental results have been collected. These running time results clearly reflect the underlying characteristics of the improved algorithm. 
Acknowledgment. We wish to express our sincere thanks to the anonymous referees for their constructive suggestions. We are grateful to Ms Chandni R. Patel for pointing out oversights in an earlier draft of this paper.

\section{References}

1. T. E. Mangir and A. Avizienis, "Fault-tolerant Design for VLSI: Effect of Interconnection Requirements on Yield Improvement of VLSI design", IEEE Trans. on Computers, vol. 31, no. 7, pp. 609-615, July 1982.

2. J.W. Greene and A.E. Gamal, "Configuration of VLSI Array in the Presence of Defects", J. ACM, vol. 31, no. 4, pp. 694-717, Oct. 1984.

3. T. Leighton and A. E. Gamal. "Wafer-scal Integration of Systoric Arrays", IEEE Trans. on Computer, vol. 34, no. 5, pp. 448-461, May 1985.

4. C.W.H Lam, H.F. Li and R. Jakakumar, "A Study of Two Approaches for Reconfiguring Fault-tolerant Systoric Array", IEEE Trans. on Computers, vol. 38, no. 6, pp. 833-844, June 1989.

5. I. Koren and A.D. Singh, "Fault Tolerance in VLSI Circuits", Computer, vol. 23, no. 7, pp. 73-83, July 1990.

6. Y.Y. Chen, S.J. Upadhyaya and C. H. Cheng, "A Comprehensive Reconfiguration Scheme for Fault-tolerant VLSI/WSI Array Processors", IEEE Trans. on Computers, vol. 46, no. 12, pp. 1363-1371, Dec. 1997.

7. T. Horita and I. Takanami, "Fault-tolerant Processor Arrays Based on the 1.5track Switches with Flexible Spare Distributions", IEEE Trans. on Computers, vol. 49, no. 6, pp. 542-552, June 2000.

8. S.Y. Kuo and W.K. Fuchs, "Efficient Spare Allocation for Reconfigurable Arrays", IEEE Design and Test, vol. 4, no. 7, pp. 24-31, Feb. 1987.

9. C.L. Wey and F. Lombardi, "On the Repair of Redundant RAM's", IEEE Trans. on CAD of Integrated Circuits and Systems, vol. 6, no. 2, pp. 222-231, Mar. 1987.

10. M.G. Sami and R. Stefabelli. "Reconfigurable Architectures for VLSI Processing Arrays", Proc. IEEE, vol. 74, no. 5, pp. 712-722, May 1986.

11. R. Negrini, M.G. Sami and R. Stefanelli, Fault tolerance through reconfiguration in VLSI and WSI arrays. The MIT Press, 1989

12. F. Distante, M.G. Sami and R. Stefanelli, "Harvesting through array partitioning: a solution to achieve defect tolerance Defect and Fault Tolerance in VLSI Systems", Proc. 1997 IEEE International Symp. Defect and Fault Tolerance in VLSI Systems, Paris, pp. 261-269, 1997

13. S.Y. Kuo and I.Y. Chen, "Efficient Reconfiguration Algorithms for Degradable VLSI/WSI Arrays." IEEE Trans. on Computer-Aided Design, vol. 11, no. 10, pp. 1289-1300, Oct. 1992.

14. C.P. Low and H.W. Leong, "On the Reconfiguration of Degradable VLSI/WSI Arrays", IEEE Trans. on Computer-Aided Design of Integrated Circuits and Systems, vol. 16, no. 10, pp. 1213-1221, Oct. 1997.

15. C.P. Low, "An Efficient Reconfiguration Algorithm for Degradable VLSI/WSI Arrays", IEEE Trans. on Computers, vol. 49, no. 6, pp. 553-559, June 2000. 